\section{Freqüência de testagem rápida para o HIV durante a admissão para o parto em puérperas no Instituto Materno Infantil Prof. Fernando Figueira, IMIP}

\section{HIV testing prevalence during antenatal care in women admitted for delivery at the Instituto Materno Infantil Professor Fernando Figueira, IMIP}

Maria Celina Rocha Morimura 1 Manoella Dantas Costa Mendes 2 Ariani Imperi de Souza 3 Luiz Cláudio Arraes de Alencar 4

1-4 Grupo Saúde da Mulher. Departamento de Pesquisa. Instituto Materno Infantil Prof. Fernando Figueira, IMIP. Rua dos Coelhos, 200. Recife, Pernambuco, Brasil. CEP: 50.070 -550

\begin{abstract}
Objectives: to determine HIV testing prevalence during antenatal care and hospital admission for delivery and identify factors associated to pregnant women testing and results delivery.

Methods : a descriptive study comprised of 400 puerperal women seen at the Women Care Center of Instituto Materno Infantil Professor Fernando Figueira, IMIP.

Results: one hundred and fifty four (38.5\%) women were admitted for delivery without having received the results of the anti-HIV test. One hundred and thirty four women (87.0\%) had been prescribed a "fast HIV test" but only 130 were tested (84.5\%). The following was determined: the number of antenatal medical visits $((p<0,001)$, the number of professionals providing antenatal care and education level ( $p=0,006)$ were significantly associated to anti-HIV testing during antenatal care, but not with results delivery. Counseling and test access during antenatal care and hospital admission for delivery were significantly associated to anti-HIV testing during antenatal care as well.

Conclusions: anti-HIV testing prevalence during antenatal care and/or screening was satisfactory in quantitative terms, however, results delivery during antenatal care, admission for delivery, and post-test counseling fell short of recommendations.
\end{abstract}

Key words Prenatal care, Disease transmission vertical, Acquired Immunodeficiency Syndrome, Crosssectional studies

\section{Resumo}

Objetivos: determinar a prevalência da testagem anti-HIV no pré-natal e internamento para o parto $e$ identificar fatores associados à realização e recebimento do resultado dos testes em parturientes.

Métodos: estudo descritivo, com 400 puérperas atendidas no Centro de Atenção à Mulher do IMIP Instituto Materno Infantil Prof. Fernando Figueira, IMIP.

Resultados: chegaram à admissão para o parto sem o resultado do teste anti-HIV, 154 (38,5\%) mulheres. Dessas, foi solicitado "teste rápido" em 134 (87,0\%), porém só foram colhidos em 130 (84,5\%). Observou-se que: o número de consultas realizadas no pré-natal, ( $p=0,001)$ o número de profissionais que atenderam a mulher no pré-natal e a escolaridade $(p=0,006)$ tiveram associação significativa com a realização do exame antiHIV no pré-natal, mas não com o recebimento do mesmo. $O$ aconselhamento e oferecimento do teste tanto no prénatal como na admissão para o parto também apresentaram associação significativa com a realização do antiHIV durante o pré-natal.

Conclusões: a prevalência de testagem anti-HIV no pré-natal elou na triagem foi satisfatório no aspecto quantitativo, porém o recebimento do resultado tanto no pré-natal quanto na admissão para o parto, bem como o aconselhamento pós-teste, foi aquém do recomendado. Palavras-chave Cuidado pré-natal, Transmissão vertical de doença, Síndrome de Imunodeficiência Adquirida, Estudos transversais 


\section{Introdução}

Estima-se que 50\% da população vivendo com HIV / AIDS em todo mundo sejam mulheres. ${ }^{1} \mathrm{O}$ baixo nível de instrução, os costumes e as normas sexuais impostas às mulheres, assim como a falta de oportunidades econômicas e de controle nos relacionamentos colaboram com a vulnerabilidade feminina para a infecção.2,3 A América Latina ocupa o quarto lugar em adultos contaminados, onde existe uma predominância da transmissão sexual (90\% dos casos novos) nas populações de comportamento de risco (usuários de drogas injetáveis e homem que faz sexo com homem) e 1,4 milhões de indivíduos vivem com HIV/AIDS, dos quais $30,0 \%$ são mulheres. O Brasil se situa entre os quatro países do mundo com o maior número de casos de AIDS notificados.1,4,5 O número de casos de AIDS notificados no período de 2000 a 2003, em Pernambuco e Recife, totalizou respectivamente 2625 e 986 casos em crescimento progressivo. 6

A partir de 1996, quando o Ministério da Saúde introduziu no Brasil a política de distribuição de medicamentos anti-retrovirais, de forma ampla e gratuita, a taxa de mortalidade por AIDS começou a apresentar queda significativa, variando de acordo com o sexo, área geográfica e nos diferentes graus de escolaridade e categorias de exposição. Desde 1999, essa taxa tem apresentado uma tendência a estabilização, com média de 6,3 óbitos por 100.000 habitantes nos últimos anos.7,8

A transmissão materno-infantil do HIV durante a gravidez e sobretudo durante o parto pode provocar danos vultosos para o bebê por ocorrer num momento de maturação do sistema imunológico. ${ }^{9}$ Atualmente, estima-se que a prevalência de infecção entre as gestantes no Brasil esteja entre $0,5 \%$ e $1 \%$. Em relação à prevenção da transmissão materno-infantil do HIV, é sabido que fazer o diagnóstico da infecção neste período possibilita a utilização de medidas preventivas que, quando bem aplicadas podem reduzir drasticamente as taxas de transmissão. ${ }^{8}$ Uma vez que isso não ocorra, ainda é possível o diagnóstico no momento do parto, através do teste rápido anti-HIV possibilitando a instauração das medidas preventivas estabelecidas. No Brasil, desde 1997, se recomenda a oferta universal do teste pré-natal (rotina voluntária) que deve ocorrer na primeira consulta de pré-natal e a possibilidade de repetir o teste em situações de exposição constante ao risco ou diante de suspeita da mulher se encontrar na "janela imunológica".8 Além disso, para realização do teste antiHIV, faz-se necessário o aconselhamento pré-teste e pós-teste, não se resumindo ao oferecimento e con- sentimento do mesmo. Esse aconselhamento representa um processo de escuta ativa, individualizada, centrada no paciente, através de uma relação de confiança entre o profissional de saúde da mulher, na busca de implementação das recomendações do Consenso 2004.8,10 Recomenda-se para os testes rápidos os mesmos princípios legais relativos aos demais testes sorológicos, garantindo o sigilo mediante o consentimento verbal da paciente. ${ }^{8}$

Dados preliminares do Sistema de Informação em Saúde no Pré-Natal (SISPRENATAL) e estimativas da Coordenação Nacional de DST / AIDS revelam que a cobertura da testagem para infecção do HIV durante o pré-natal está abaixo de 40,0\%.11 Considerando ainda a baixa adesão e/ou captação tardia ao pré-natal, essa cobertura se torna ainda menor. ${ }^{11}$ Apesar da disponibilidade das intervenções profiláticas para minimizar a transmissão vertical do HIV no Brasil para toda a população, a administração de medicação para profilaxia de TMI (zidovudina injetável) ainda ocorre em menos de 50,0\% dos partos de mulheres estimadas como infectadas pelo vírus. 8 Alguns fatores podem estar relacionados a vários problemas: dificuldades da rede em prover diagnóstico laboratorial da infecção pelo HIV, cobertura insuficiente de mulheres testadas no pré-natal e qualidade do pré-natal, ainda aquém do desejável. No entanto, mesmo com essas dificuldades, o número de casos de AIDS em crianças vem diminuindo progressivamente em todo país. 8

$\mathrm{O}$ acesso à terapêutica medicamentosa para as pessoas infectadas para o HIV é uma das prioridades da saúde pública mundial, porém só o acesso às drogas anti-retrovirais não é suficiente. O objetivo deve ser buscar a combinação da prevenção com os cuidados clínicos, para que se possa interferir na progressão da epidemia que apresenta características dinâmicas e em continua transformação. ${ }^{12,13}$ Desse modo, faz-se necessário conhecer o status sorológico em que se encontram as gestantes e parturientes quanto ao diagnóstico do HIV, possibilitando a implementação das medidas profiláticas e terapêuticas disponíveis na busca de minimizar ou erradicar a TMI do HIV. ${ }^{8}$ Os resultados obtidos pelo protocolo 076 do Pediatrics Aids Clinical Trials Group (PACTG 076), em 1994, foram um marco na saúde pública. Posteriormente outros protocolos semelhantes foram testados através de ensaios clínicos randomizados, demonstrando diminuição significativa na transmissão vertical do HIV14. Novas diretrizes públicas pela WHO, mostraram a possível utilização de esquemas anti-retroirais (TARV) combinados compostos por drogas seguras para o feto e que são os indicados atualmente, evidenciando me- 
lhor eficácia na prevenção da TMI.15 Embora a testagem rápida funcione como uma última oportunidade de diagnóstico e possibilidade de utilização da TARV, inclusive durante o parto, ele representa também um fracasso parcial dos cuidados pré-natais da prevenção da TMI. No Brasil, a epidemia não está sob controle, sendo necessário fomentar a prevenção da transmissão assim como acompanhar sua evolução, inclusive as características comportamentais ou epidemiológicas dos casos, além dos diferentes padrões de transmissão nas diversas regiões do país. 16

Este estudo teve como objetivo determinar a prevalência de testagem anti-HIV e do recebimento do resultado do teste durante o pré-natal e na admissão para o parto e investigar fatores associados à realização e ao recebimento da sorologia para o HIV durante o pré-natal em parturientes admitidas numa maternidade-escola na cidade do Recife.

\section{Métodos}

Foi realizado um estudo descritivo, retrospectivo, tipo corte transversal, em 400 puérperas do Centro de Atenção à Mulher (CAM) no Instituto Materno Infantil Prof. Fernando Figueira (IMIP), localizado na cidade do Recife, Pernambuco, Brasil. O estudo foi realizado no mês de outubro de 2003. As mulheres foram selecionadas através de visitas diárias ao alojamento conjunto, onde foram esclarecidas sobre a temática e os objetivos da pesquisa. Foram excluídas as sabidamente soropositivas para o HIV antes da gestação. No estudo, considerou-se que a realização do exame anti-HIV (ELISA) fora feita no pré-natal, quando havia registro do resultado do exame no cartão de pré-natal trazido pela mulher ou no prontuário do pré-natal daquelas que haviam feito pré-natal no serviço. Os kits utilizados no serviço para o teste rápido anti-HIV, seguem as recomendações preconizadas pelo Ministério da Saúde. Para coleta de dados foi utilizado um questionário padronizado, com respostas pré-codificadas. Para investigar a associação dos fatores associados à testagem anti-HIV, utilizaram-se os testes $\chi 2$ de Pearson e $\chi 2$ de Tendência. O estudo foi aprovado pelo Comitê de Ética e Pesquisa em Seres Humanos da Instituição. Só participaram as mulheres que assinaram o termo de consentimento livre esclarecido. Para as mulheres que apresentaram resultado reagente para o HIV, bem como para os recémnascidos, foi disponibilizado o tratamento preconizado pelo Ministério da Saúde durante o internamento e após a alta.

\section{Resultados}

Das 400 mulheres do estudo, 154 (38,5\%) chegaram à triagem para o parto sem o resultado do teste antiHIV do pré-natal (Tabela 1). Destas, 134 (87,0\%) tiveram os "testes rápidos" anti-HIV solicitados pelo profissional que atendeu a mulher para o parto na triagem, no entanto apenas $130(84,5 \%)$ realizaram o exame, nesse momento. Quanto ao recebimento do resultado do teste rápido anti-HIV, 125 mulheres $(96,2 \%)$ não receberam o resultado (Tabela 2 ).

Do total das mulheres do estudo, 300 realizaram o teste anti-HIV no pré-natal. O numero de consultas pré-natal, o número de profissionais que assistiram no pré-natal, a escolaridade da mulher, estiveram estatisticamente associados $(p<0,001, p<0,001 \mathrm{e}$ $p=0,006$ respectivamente) à realização do teste anti-

Tabela 1

Distribuição de freqüência de registro na admissão para o parto do teste anti-HIV realizado no pré-natal. Centro de Atenção à Mulher do Instituto Materno Infantil Prof. Fernando Figueira - IMIP. Recife, Pernambuco, outubro 2003.

\begin{tabular}{lrr}
\hline Variáveis & N & $\%$ \\
\hline Chegou à admissão & & \\
Com resultado anti-HIV do pré-natal & 246 & 61,5 \\
Sem resultado anti-HIV do pré-natal & 154 & 38,5 \\
Total & 400 & 100,0 \\
\hline
\end{tabular}

Tabela 2

Distribuição de freqüência de solicitação e coleta do teste rápido anti-HIV, das 154 parturientes se registro de resultado do anti-HIV no pré-natal, no memento da admissão para o parto e do recebimento do resultado do teste. Centro de Atenção à Mulher do Instituto Materno Infantil Prof. Fernando Figueira - IMIP, outubro 2003.

\begin{tabular}{lrr}
\hline Variáveis & N & $\%$ \\
\hline Solicitado teste rápido anti-HIV & 134 & 87,0 \\
$\quad$ Sim & 20 & 13,0 \\
$\quad$ Não & 154 & 100,0 \\
$\quad$ Total & & \\
Colheu teste rápido anti-HIV & 130 & 84,5 \\
Sim & 24 & 15,5 \\
Não & 154 & 100,0 \\
Total & & \\
Recebeu resultado do teste rápido & 5 & 3,8 \\
Sim & 125 & 96,2 \\
Não & 130 & 100,0 \\
$\quad$ Total & & \\
\hline
\end{tabular}


Associação entre as principais características epidemiológicas e a realização ou recebimento do resultado da sorologia anti-HIV no prénatal. Centro de Atenção à Mulher do Instituto Materno Infantil Prof. Fernando Figueira - IMIP. Recife, Pernambuco, outubro 2003.

\begin{tabular}{|c|c|c|c|c|c|c|c|c|c|c|}
\hline \multirow[t]{2}{*}{ Variáveis } & \multicolumn{4}{|c|}{ Colheu } & \multirow[t]{2}{*}{$p^{*}$} & \multicolumn{4}{|c|}{ Recolheu } & \multirow[t]{2}{*}{$p^{*}$} \\
\hline & Sim & $\%$ & Não & $\%$ & & Sim & $\%$ & Não & $\%$ & \\
\hline \multicolumn{11}{|c|}{ Número de consultas pré-natal } \\
\hline Até 3 & 36 & 12,0 & 23 & 28,8 & $<0,001$ & 27 & 11,2 & 8 & 14,3 & 0,050 \\
\hline $4-6$ & 129 & 43,0 & 41 & 51,2 & & 98 & 40,5 & 31 & 55,3 & \\
\hline$>6$ & 135 & 45,0 & 16 & 20,0 & & 117 & 48,3 & 17 & 30,4 & \\
\hline Total & 300 & 100,0 & 80 & 100,0 & & 242 & 100,0 & 56 & 100,0 & \\
\hline \multicolumn{11}{|c|}{ Número de profissionais que a assistiram no pré-natal } \\
\hline $1-2$ & 156 & 52,0 & 72 & 90,0 & $<0,001$ & 130 & 53,7 & 25 & 44,6 & 0,281 \\
\hline Acima de 3 & 144 & 48,0 & 8 & 10,0 & & 112 & 46,3 & 31 & 55,4 & \\
\hline Total & 300 & 100,0 & 80 & 100,0 & & 242 & 100,0 & 56 & 100,0 & \\
\hline \multicolumn{11}{|l|}{ Idade } \\
\hline $13-19$ & 83 & 27,7 & 23 & 28,7 & 0,050 & 73 & 30,1 & 10 & 17,9 & 0,179 \\
\hline $20-29$ & 155 & 51,6 & 31 & 38,7 & & 120 & 49,6 & 33 & 58,9 & \\
\hline $30-44$ & 62 & 20,7 & 26 & 32,6 & & 49 & 20,3 & 13 & 23,2 & \\
\hline Total & 300 & 100,0 & 80 & 100,0 & & 242 & 100,0 & 56 & 100,0 & \\
\hline \multicolumn{11}{|l|}{ Escolaridade } \\
\hline Nenhum & 12 & 4,0 & 5 & 6,3 & 0,006 & 9 & 3,8 & 2 & 3,6 & 0,954 \\
\hline $1-3$ anos & 18 & 6,0 & 11 & 13,7 & & 14 & 5,8 & 4 & 7,2 & \\
\hline 4-7 anos & 105 & 35,0 & 36 & 45,0 & & 86 & 35,5 & 18 & 32,1 & \\
\hline 8 ou mais anos & 165 & 55,0 & 28 & 35,0 & & 133 & 54,9 & 32 & 57,1 & \\
\hline Total & 300 & 100,0 & 80 & 100,0 & & 242 & 100,0 & 56 & 100,0 & \\
\hline
\end{tabular}

*Teste $\chi^{2}$ de tendência.

Tabela 4

Associação entre o aconselhamento e / ou o oferecimento pré-teste anti-HIV com a realização da sorologia no prénatal. Centro de Atenção à Mulher do Instituto Materno Infantil Prof. Fernando Figueira - IMIP. Recife, Pernambuco, outubro 2003.

\begin{tabular}{|c|c|c|c|c|c|}
\hline \multirow[t]{3}{*}{ Variáveis } & \multicolumn{4}{|c|}{ Realização do teste anti-HIV pré-natal } & \multirow[t]{3}{*}{$p^{*}$} \\
\hline & \multicolumn{2}{|c|}{ Sim } & \multicolumn{2}{|c|}{ Não } & \\
\hline & $\mathrm{n}$ & $\%$ & $\mathrm{n}$ & $\%$ & \\
\hline \multicolumn{6}{|c|}{ Aconselhamento pré-teste no pré-natal } \\
\hline Sim & 143 & 47,7 & 18 & 22,5 & $<0,001$ \\
\hline Não & 155 & 51,8 & 62 & 77,5 & \\
\hline Não sabe informar & 2 & 0,5 & - & - & \\
\hline Total & 300 & 100,0 & 80 & 100,0 & \\
\hline \multicolumn{6}{|c|}{ Oferecimento do teste no pré-natal } \\
\hline Sim & 115 & 38,4 & 18 & 22,5 & 0,010 \\
\hline Não & 183 & 61,1 & 62 & 77,5 & \\
\hline Não sabe informar & 2 & 0,5 & - & - & \\
\hline Total & 300 & 100,0 & 80 & 100,0 & \\
\hline
\end{tabular}

*Teste $\chi^{2}$ de tendência. 
Associação entre o aconselhamento e o a oferecimento para o teste rápido anti-HIV com a realização do teste rápido na triagem. Centro de Atenção à Mulher do Instituto Materno Infantil Prof. Fernando Figueira (IMIP). Recife, Pernambuco, outubro 2003.

\begin{tabular}{|c|c|c|c|c|c|}
\hline \multirow[t]{3}{*}{ Variáveis } & \multicolumn{4}{|c|}{ Realização do teste anti-HIV pré-natal } & \multirow[t]{3}{*}{$p^{*}$} \\
\hline & \multicolumn{2}{|c|}{ Sim } & \multicolumn{2}{|c|}{ Não } & \\
\hline & $\mathrm{n}$ & $\%$ & $\mathrm{n}$ & $\%$ & \\
\hline \multicolumn{6}{|l|}{ Aconselhamento para teste rápido anti-HIV } \\
\hline Sim & 17 & 7,7 & - & - & $<0,001$ \\
\hline Não & 201 & 90,5 & 171 & 96,1 & \\
\hline Não sabe informar/feto morto/inconsciente & 4 & 1,8 & 7 & 4,0 & \\
\hline Total & 222 & 100,0 & 178 & 100,0 & \\
\hline \multicolumn{6}{|l|}{ Oferecimento do teste rápido anti-HIV } \\
\hline Sim & 29 & 13,1 & - & - & $<0,001$ \\
\hline Não & 188 & 84,6 & 171 & 96,1 & \\
\hline Não sabe informar/feto morto/inconsciente & 5 & 2,3 & 7 & 4,0 & \\
\hline Total & 222 & 100,0 & 178 & 100,0 & \\
\hline
\end{tabular}

*Teste $\chi^{2}$ de tendência.

HIV durante o pré-natal, mas não ao recebimento do resultado dos testes (Tabela 3). Das 300 parturientes que colheram o teste anti-HIV durante o pré-natal, $155(51,8 \%)$ não fizeram aconselhamento pré-teste para $183(61,1 \%)$ o pré-natalista não ofereceu a testagem (Tabela 4$)$.

Observou-se (Tabela 5) que das 222 parturientes que realizaram a testagem rápida para o HIV na triagem, apenas $17(7,7 \%)$ receberam aconselhamento pré-teste e a $29(13,1 \%)$ delas foi oferecido o exame pelo profissional na admissão para o parto. $\mathrm{O}$ aconselhamento e o oferecimento estiveram estatisticamente associados tanto à realização do teste anti-HIV no pré-natal quanto à realização do "teste rápido" na triagem. Em relação aos resultados da testagem anti-HIV, cinco testes foram reagentes no pré-natal e um na triagem, sendo que este último resultou em "não-reagente" no teste confirmatório posterior.

\section{Discussão}

Apesar da disponibilidade do teste anti-HIV na assistência pré-natal da rede pública no Brasil desde 1998, 38,5\% das mulheres do estudo chegaram à triagem sem o resultado do teste, seja porque não realizaram o pré-natal, não colheram o exame no pré-natal ou não receberam o resultado do teste reali- zado. Embora a testagem rápida anti-HIV no momento da admissão para o parto representem a última oportunidade diagnóstica para essa gestação, 24 das 400 (6\%) mulheres pariram sem a realização do teste anti-HIV em nenhum desses momentos. Desse modo, observou-se que a efetividade das ações para profilaxia da TMI do HIV ainda está aquém do recomendado. Essa situação é agravada quando consideramos que o ato de coleta não garante à mulher o recebimento do resultado que permitiria o conhecimento de seu status sorológico para HIV e conseqüentemente propiciaria intervenções para reduzir ou até erradicar as chances de transmissão materno-infantil do vírus HIV. ${ }^{8}$ Apesar de um alto percentual $(38,5 \%)$ de mulheres terem chegado à triagem para o parto sem o resultado da testagem do pré-natal, o teste rápido só foi colhido em 84,5\% delas (24 mulheres). Perdeu-se, dessa forma, a ultima oportunidade para testagem e as intervenções preventivas da TMI. Vale destacar o alto nível de aceitação pelas gestantes da testagem anti-HIV referido na literatura. ${ }^{17,18}$ Ressalta-se que o Ministério da Saúde preconiza a realização da testagem anti-HIV na primeira consulta do pré-natal e ao mesmo tempo viabiliza a sorologia, o que permitiria encontrar uma cobertura próxima a $100,0 \%$ da testagem nas gestantes. 8 A meta do Ministério da Saúde para o ano de 2007 é reduzir a quase zero a infecção do HIV em recém-nascidos. 19 
Isto leva à indagação sobre a qualidade da assistência prestada no pré-natal e a atenção dada pelo profissional à prevenção da TMI. Sob o prisma do profissional, as recomendações do Ministério da Saúde sobre a TMI do HIV, ganham força de lei pela obrigatoriedade de oferecimento do exame anti-HIV no pré-natal, segundo determinação do Conselho Federal de Medicina em maio de 2003.20 De acordo com o Código de Ética de Enfermagem, o enfermeiro tem autonomia para acompanhar a gestante de baixo risco durante o pré-natal, podendo solicitar os exames de rotina e complementares de acordo com os Programas do Ministério da Saúde. ${ }^{21}$ A ausência de teste anti-HIV no pré-natal e durante o internamento para o parto, revela que a efetividade da prevenção da TMI ainda não está assegurada a todas as mulheres nos serviços de saúde.

O percentual de mulheres que chegaram para o parto com o resultado do teste anti-HIV do pré-natal $(61,5 \%)$, apesar de comparável a outros estudos, 22 ainda é considerado baixo, pela repercussão que pode representar para saúde do filho, bem como para a saúde pública. Essa baixa prevalência de testagem tem sido referida especialmente nas regiões Norte e Nordeste do Brasil onde se destacam as falhas na assistência pré-natal como fator que influencia o alto número de vítimas da TMI do HIV.23 Estudos realizados no serviço obstétrico público no Rio Grande do Sul evidenciaram que das 298 gestantes avaliadas, 168 haviam recebido assistência pré-natal e dessas 30,5\% afirmaram terem sido testadas previamente, embora não dispusessem de resultado no momento da admissão na maternidade. Os autores recomendaram a necessidade de treinamento da equipe a fim de estimular as pacientes durante o prénatal a portarem a documentação específica (cartão da gestante) na internação para o parto, possibilitando à equipe que vai assistir o parto ter ciência da situação da gestante durante o pré-natal.24 Questiona-se o quanto a taxa de prevalência de testagem está relacionada à preocupação do prénatalista ou da organização administrativa do serviço. Podemos considerar que a disseminação da testagem anti-HIV como um direito da paciente no pré-natal encontra-se em fase de expansão e que existe uma estruturação das políticas de saúde do Governo Federal. No entanto, o funcionamento dos sistemas de referência e contra-referência dos serviços de saúde com os laboratórios que realizam os exames, os fluxos de coleta dos exames, dentre outros fatores, devem ser reavaliados de modo a contribuir para o diagnóstico precoce do HIV. O fato de que a solicitação do teste observada neste trabalhos não foi feita durante o internamento em 20 dos
154 casos que não possuíam resultado do teste antiHIV pré-natal, sugere que fatores como a alta demanda do serviço onde o estudo foi desenvolvido, bem como a dificuldade para os profissionais em esclarecer sobre uma temática repleta de imaginário e preconceitos em informar a positividade à gestante, além de possível minimização da preocupação com o problema, tenham interferido no oferecimento do exame. Além disso, em quatro situações em que o exame foi solicitado, a coleta foi feita, totalizando $15,5 \%$ de parturientes sem o conhecimento de sua situação sorológica para o HIV no momento do parto. A ausência da coleta é outro fato que pode estar relacionado à dificuldade na capacidade do serviço de atender a alta demanda, incluindo os casos das pacientes que chegam em "período expulsivo". Quanto ao recebimento do resultado do teste rápido para o $\mathrm{HIV}$, foi referido por apenas cinco $(3,8 \%)$ das mulheres que realizaram o exame. Essa baixa taxa indica que a rotina do serviço deveria incorporar a busca ativa de todos os exames solicitados. A responsabilidade da busca do exame cabe ao profissional que o solicitou. 20 Observou-se, ainda, que o número de consultas de pré-natal foi associado a uma maior realização de exames antiHIV durante esse período. Das 59 pacientes que tiveram até três consultas de pré-natal, apenas 36 colheram o exame, enquanto que das 151 que receberam mais de seis atendimentos, 135 tiveram o exame colhido no pré-natal. O Ministério da Saúde considera a realização de pré-natal quando a paciente freqüenta no mínimo seis consultas. ${ }^{8}$ Essa situação ideal, não corresponde, no entanto, à aqui abordada. Para a testagem anti-HIV o número aumentado de consultas mostra melhores e mais claras chances de coleta do exame. Diante do número insuficiente de consultas em muitas gestantes é importante ressaltar a necessidade do profissional avaliar cada consulta pré-natal como oportunidade única e maximizar a atenção para cada uma delas. Em relação ao número de profissionais, os dados revelaram que as pacientes que tiveram atendimento com mais de três profissionais diferen-tes nas consultas tiveram maior chance com a rea-lização da coleta da testagem, porém o mesmo não ocorreu com a chance do recebimento, que não se relacionou ao número de profissionais. Esse fato parece paradoxal à qualidade na assistência, já que se considera importante para a continuidade da assistência à mulher que o mesmo profissional avalie sua evolução gestacional, progrida em complexidade de informações relativas aos cuidados com a gestação, orientações para o trabalho de parto, parto propriamente dito e puerpério, permitindo manter uma relação de confiança 
entre a paciente-familiares-profissionais e serviços de saúde. Observou-se, quanto ao aspecto especifico da coleta do anti-HIV no pré-natal, que o maior número de profissionais permite melhores condições para a mulher conseguir realizar a testagem durante o pré-natal, pelo fato de que nem todos os profissionais estão sensibilizados a prestar a assistência em saúde dentro da regulamentação legal exigida. Além disso, a informação em saúde ainda não está adequada, a fim de propiciar a exigência da mulher para essa testagem específica. Vale ressaltar toda a contextualização que envolve o HIV, que afeta os profissionais e as pacientes, como o medo, o preconceito, os tabus e os valores individuais. Houve evidência de associação entre a idade das mulheres e a realização do exame no pré-natal. A escolaridade esteve fortemente associada à coleta porém não ao recebimento do teste.Isso pode indicar que as mulheres mais esclarecidas têm mais cuidados com a realização do exame, porém o recebimento pode estar ligado a fatores que não dependem delas. Os achados mostram a importância da relação entre a educação, a saúde e o desenvolvimento social. Das 300 mulheres que realizaram o teste anti-HIV no pré-natal, mais da metade referiram não terem sido aconselhadas pelo profissional antes da solicitação. No que se refere ao oferecimento do teste, $61,1 \%$ afirmaram não ter sido oferecido pelo profissional o exame. A testagem anti-HIV é universal e voluntária no Brasil, porém tanto o aconselhamento quanto oferecimento estiveram diretamente associados à realização do teste no pré-natal. Dessa forma, toda a pessoa para a qual seja necessária a solicitação do teste, deve ter obrigatoriamente garantido o aconselhamento, o oferecimento e o consentimento informado para a realização do exame, assegurando-se o acesso e o sigilo. ${ }^{8} \mathrm{O}$ pré-natalista deveria atuar em cada consulta como se estivesse diante da única chance de oferecer à mulher a testagem, para que ela tivesse oportunidade de conhecer o seu status sorológico para o HIV e proporcionar a profilaxia da TMI para o seu filho. Em situações em que a gestante não retorna ao pré-natal após a realização do teste, o serviço de saúde deveria estar preparado para realizar a busca dessa paciente, de modo universal. Na impossibilidade dessa condição a todas as mulheres, ao menos àquelas que apresentam sorologia anti-HIV reagente. Vale ressaltar ainda a importância de redução do tempo entre a testagem e o conhecimento do resultado pela gestante no prénatal, através de um "fluxo diferencial". Quanto ao intervalo de tempo entre a solicitação e o resultado, praticamente $80 \%$ das gestantes receberam o resultado em média 30 dias após a coleta. Esse período de tempo parece razoável para o resultado, se considerarmos a solicitação precoce do exame. A testagem rápida para o HIV não deve ser realizada sem um aconselhamento detalhado acompanhado das informações pertinentes ao assunto, assim como já comentado acima sobre a testagem convencional. No entanto constatou-se um número alto $(90,5 \%)$ de testagem rápida sem esse devido cuidado. Do total de mulheres que receberam o resultado da testagem no pré-natal, $5(1,7 \%)$ obtiveram resultados "reagentes" e foram admitidas na triagem conhecedoras de seu status sorológico, possibilitando os cuidados para minimizar o risco de contaminação do concepto. Das 222 mulheres que foram testadas durante o internamento na triagem obstétrica, $1(0,3 \%)$ foi "reagente", sendo que o exame confirmatório realizado posteriormente afastou a "soro-positividade" para o HIV. Embora se considere atualmente que o teste rápido tenha tanta confiabilidade quanto os exames sorológicos utilizados no pré-natal, ainda é indispensável a realização do teste confirmatório.

\section{Conclusões}

O estudo possibilitou uma visão sobre o conhecimento do status sorológico para o HIV e seus possíveis fatores associados, além da identificação de características epidemiológicas em parturientes admitidas para o parto num hospital-escola da cidade do Recife. Dentre as formas de transmissão do HIV a materno-infantil é a que possibilita maior diminuição da transmissão dessa infecção e a única passível de erradicação completa. Os outros meios de transmissão não apresentam essa mesma possibilidade.

A prevalência da testagem anti-HIV no pré-natal e/ou na triagem foi satisfatória no aspecto quantitativo, porém o recebimento do resultado, tanto no pré-natal, quanto na admissão para o parto, bem como o aconselhamento pós-teste, foi aquém do recomendado.

Esses dados reforçam a necessidade de uma melhoria urgente dos fluxos clínicos - laboratoriais para diminuir a TMI.

\section{Agradecimentos}

Ao Instituto Materno Infantil Prof. Fernando Figueira, IMIP pelo suporte e ao Conselho Nacional de Desenvolvimento Científico e Tecnológico, CNPQ pela bolsa do Programa de Iniciação Científica. 


\section{Referências}

1. Rapparini C. Transmissão do HIV In: Fórum Científico HIV/AIDS. [on line]. Disponível em: URL: http:www.hiv.org.br. [2004 mar 23]

2. Herrera C, Campera L. La vulnerabilidad e invisibilidad de las mujeres ante el VIH/SIDA: constantes y câmbios em el tema. Salud Pub Mex. 2002, 44: 555-64.

3. Ministério da Saúde. Coordenação Nacional de DST e AIDS. Ministério da Saúde divulga novos dados da AIDS e anuncia resistência do HIV aos anti-retrovirais [on line]. Brasília (DF), 2003. Disponível em: URL:http:// www.aids.gov.br/final/imprensa1/dados.htm. [2004 nov 15].

4. UNAIDS. Report on the global AIDS epidemic 2004 [on line].. Disponível em: URL: http://www.unaids.org/Unaids/ EN/. [2004 nov 15].

5. Ministério da Saúde. Secretaria de Projetos Especiais de Saúde. Introdução [on line]. Disponível em: URL:http:// www.aids.gov.br/2000. [Acesso].

6. Ministério da Saúde. Dados e esquisas em DST e AIDS 2003 [on line]. Disponível em URL: http:// www.aids.gov.br/final/dados/aids.htm . [2004 nov 15].

7. Szwarcwald CL, Bastos FI, Esteves MAP, Andrade CLT. A disseminação da epidemia da AIDS no Brasil, no período de 1987-1996: uma análise espacial. Cad Saúde Publica. 2000; 16: 7-19.

8. Ministério da Saúde. Coordenação Nacional de Doenças Sexualmente Transmissíveis/Aids. Recomendações para profilaxia da transmissão vertical do HIV e terapia antiretroviral em gestantes. Brasília (DF); 2003.

9. Ortigão-de-Sampaio MB, Castello Branco LRR. Imaturidade imunológica fetal e neonatal: implicações na evolução clínica da infecção pelo HIV-1 em crianças. Rev Assoc Med Bras. 1997; 47: 29-34.

10. Simpson WM, Jonhstone FD, Boyd FM, Goldberg DJ, Hart GJ, Prescott RJ. Uptake and acceptability of antenatal HIV testing: randomised controlled tril of different methods of offereing the test. Br Med J. 1998; 316: 262-7.

11. Brasil. Portaria n. 2.104, de 19 de novembro de 2002. Regulamenta Projeto Nascer-Maternidades. Diário Oficial da União. [DOU] 2002 nov 21 (225).

12. Reynolds SJ, Bartlett JG, Quinn TC, Beyrer C, Bollinger RC. Antiretroviral therapy where resources are limited. N Engl J Med. 2003; 348: 1806-9.

13. Castilho EA, Bastos FI. Aids (1981-97): o rastro da tormenta. Rev USP: Dossiê Aids. 1997; 33: 8-19.
14. Gray G, McIntyre J, Mmiro F, Ndugwa Ch, Coovadia HM, Moodley J, Kilewo Ch, Massawe A, Kituuka P, Okong P. Efficacy of three short-course regimens of zidovudine and lammivudine in preventing early and late transmission of HIV-1 from mother to child in Tanzânia, South África, and Uganda (Petra Study): randomized, doublé-blinde, placebocontrolled trial. Lancet. 2002; 359: 1178-86.

15. WHO (World Health Organization). WHO publics new guidelines on preventing mother to children transmission of HIV [on line]. Disponível em URL:http:// www.who.int/ 3by5/news17/en/ [2004 out 26].

16. Vermelho LL, Silva LP, Costa AJL. Epidemiologia da transmissão vertical do HIV no Brasil [on line]. In: Ministério da Saúde. Coordenação Nacional de DST e AIDS. Disponível em URL: www.aids.gov.br/udtv/ boletim_jun_ago99/trans_vertical.htm. [2004 nov 2004].

17. Goldani MZ, Giurgliane ERJ, Scanlon T, Rosa H, Castilhos K, Feldens L, Tomkins A. Voluntary HIV counseling and testing during prenatal care in Brazil. Rev Saúde Pública. 2003; 37: 552-8.

18. Schuman P, Jones TB, Ohmit S, Marbury C, Moore J, Marilyn P. Voluntary HIV counseling and testing of pregnancy women - an assessment of compliance with Michigan Public Health Statutes. Med Gen Med. 2004; 6 (2).

19. Ministério da Saúde. Secretaria de Vigilância em Saúde. Departamento de Analise de Situação em Saúde. Brasil 2004: uma analise da situação de saúde. Brasília (DF); 2004.

20. Conselho Federal de Medicina. Resolução CFM n ${ }^{\circ} 1665$, de 7 de Maio de 2003. Diário Oficial da União. [DOU] 2003; 3 jun, Sec 1: 83-4.

21. Conselho Federal de Enfermagem. Resolução COFEN195/1997 [on line]. Disponível em: URL:www.portalcofen.com.br. [2004 dez. 12].

22. Neves FR, Passos ADC, Gueeleri WL. Disponbilidade de sorologia anti-HIV como um teste voluntário na rotina do atendimento pré-natal em unidades básicas de saúde. Rev Saúde Pública. 1999; 33: 624-5.

23. Cavalcante MS, Ramos Junior NA, Jesus e Silva TM, Pontes LRSK. Transmissão vertical do HIV em Fortaleza: revelando a situação epidemiológica em uma capital do Nordeste. Rev Bras Ginecol Obstet. 2004; 26: 131-8.

24. Carvalho RL, Krahe C, Farina G, Paula DO, Richetti N, Crosseti T. Teste rápido para diagnóstico da infecção pelo HIV em parturientes. Rev Bras Ginecol Obstet. 2004; 26 : 325-8.

Recebido em 7 de novembro de 2005

Versão final apresentada em 25 de maio de 2006

Aprovado em 3 de abril de 2006 